\title{
Where to Predict the Channel for Cooperative Multi-Cell Transmission over Correlated Subcarriers?
}

\author{
Richard Fritzsche, Eckhard Ohlmer, Gerhard P. Fettweis \\ Technische Universität Dresden, Vodafone Chair Mobile Communications Systems, Dresden, Germany \\ Email: \{richard.fritzsche, eckhard.ohlmer, fettweis\}@ifn.et.tu-dresden.de
}

\begin{abstract}
In this work we discuss the aspect of channel prediction for cooperative multi-cell downlink transmission, where channel state information (CSI) of all users need to be available at all cooperating base stations (BSs). We assume that users feed CSI back to its local BS which forwards it to the other cooperating BSs using backhaul connections. In case of feedback and backhaul latency, CSI of a single user equipment (UE) is affected by multiple delays. Compensating for the delay via channel prediction raises the question of where to place the predictor. Prediction at the UE before the channel observations are quantized allows to compensate only for a single delay. Prediction at the BS side keeps the flexibility to compensate for the actual delay at each base station, at the drawback that less accurate information is available due to feedback quantization. This paper extends previous work from a transmission over uncorrelated subcarriers to the more realistic transmission over correlated subcarriers. Previously, we have shown that prediction before and after quantization results in the same channel uncertainty. As a consequence, prediction at the BS is always preferable if multiple delays need to be compensated. This paper shows that this result remains valid also for correlated subcarriers.
\end{abstract}

\section{INTRODUCTION}

Cooperation between base stations (BSs) in cellular communication networks may in theory lead to substantial gains in terms of user throughput compared to non-cooperative techniques [1]-[3]. In the downlink, inter-cell and inter-user interference can be shaped beneficially by deploying joint precoding techniques, which implement a pre-equalization of the user data, based on channel state information (CSI) [4], [5]. The CSI quality at the BSs has a significant impact on the user performance [6]. Optimizing the CSI accuracy is, therefore, of major interest [7], [8]. In addition, robust precoding techniques can be used to compensate for remaining CSI inaccuracies [5], [9], [10]. In this work, we focus on distributed precoding, where CSI of all jointly precoded user equipments (UEs) needs to be available at all cooperating BSs [11], [12]. In contrast, centralized precoding requires CSI of all UEs to be available at a central node $(\mathrm{CN})$, where the processing is performed (see Fig. 1) [13]. Regarding a frequency devision duplex (FDD) system, a UE feeds CSI back to its local BS which forwards the CSI to the other BSs. For such a system, three sources of CSI impairments can be identified: noisy pilot reception, CSI quantization at the UE and feedback/backhaul delays [14]. From a BS perspective, CSI of other cell UEs is more outdated compared to CSI of local UEs, due to backhaul latency. Delay

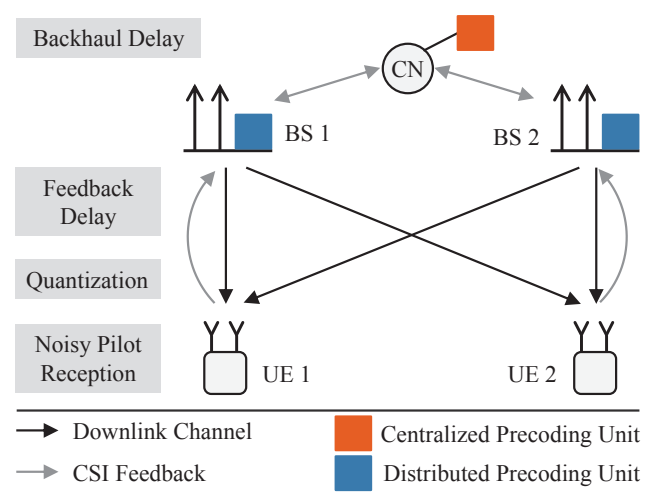

Fig. 1. Centralized and distributed precoding with impaired CSI.

based CSI inaccuracies can be reduced by employing channel prediction techniques. In this paper, we discuss the basic question of where to predict the channel if CSI is affected by multiple delays. Prediction at the UE side can only compensate for a single delay. In contrast, prediction at the BS side keeps the flexibility to predict for the actual delay at each BS, while less accurate CSI is available due to feedback quantization. The paper is a generalization of our previous work [14], where we found that prediction before and after quantization results in the same channel uncertainty. Consequently, prediction at the BS rather than at the UE is preferable for distributed precoding. However, this result has been derived under the assumption of CSI coupled to uncorrelated subcarriers. In this paper, we extend our findings to the more general case of correlated subcarriers.

The remainder of this manuscript is structured in the following way. The system model for CSI feedback is introduced in Section II, while in Section III the different prediction options are presented. In Section IV exemplary results are shown followed by conclusions in Section V.

Notation: Conjugate, transposition and conjugate transposition is denoted by $(\cdot)^{*},(\cdot)^{T}$ and $(\cdot)^{H}$, respectively. The trace of a matrix is written $\operatorname{as} \operatorname{tr}(\cdot), \operatorname{diag}(\cdot)$ creates a diagonal matrix out of a column vector, while $\operatorname{diag}^{-1}(\cdot)$ stacks the diagonal elements of a matrix into a column vector. $\mathbb{E}\{\cdot\}$ denotes expectation, $\mathbb{C}$ is the set of complex numbers and $\mathcal{N}_{\mathbb{C}}(\mathbf{m}, \boldsymbol{\Phi})$ refers to a multi-variate complex normal distribution with mean vector $\mathbf{m}$ and covariance matrix $\boldsymbol{\Phi}$. 


\section{SySTEM MODEL}

In this section we introduce the mathematical model for CSI feedback. Later on, the performance is evaluated based on the average user mean square error (MSE) between the actual channel and the CSI used for precoding at the BS.

\section{A. Downlink Channel Model}

For modeling the channel in downlink direction we assume orthogonal-frequency-devision-multiplexing, where orthogonal symbols are transmitted in a time and frequency grid, with symbol rate $f_{T}$ and subcarrier spacing $f_{F}$. It is assumed that the channel is static during the transmission of $L_{T}$ symbols in time and $L_{F}$ symbols in frequency. We define a transmission block as the collection of $L=L_{T} L_{F}$ symbols experiencing the same channel state $h[t, f] \sim \mathcal{N}_{\mathbb{C}}(0, \lambda)$, where $t$ and $f$ is the block index in time and frequency, respectively. The mean channel gain reads

$$
\lambda=\beta d^{-\alpha}
$$

with path loss exponent $\alpha$, distance $d$ between UE and BS as well as coefficient $\beta$ to further adjust the model. Note, that each BS-UE connection has an individual channel gain. We consider a block-static time-varying and frequency selective channel, where two blocks at different time and frequency are statistically correlated depending on the user velocity $v_{u}$ and the maximum delay spread $\tau$ [15]. Based on a Jakes Doppler spectrum and a normalized delay $\Delta$ the covariance in time is

$$
\mathbb{E}\left\{h[t, f] h^{*}[t \pm \Delta, f]\right\}=c_{T}[\Delta]=J_{0}\left(2 \pi \frac{L_{T} f_{C} v_{u}}{f_{T} v_{c}} \Delta\right) \lambda,
$$

where $f_{C}, c$ and $J_{0}$ are the carrier frequency, the speed of light and the zero-th order Bessel function of the first kind, respectively. With $\operatorname{si}(x)=\sin (x) / x$ the covariance in frequency of two blocks with distance $\Delta$ is denoted as

$$
\mathbb{E}\left\{h[t, f] h^{*}[t, f \pm \Delta]\right\}=c_{F}[\Delta]=\operatorname{si}\left(2 \pi \tau L_{F} f_{F} \Delta\right) \lambda .
$$

Note, that our modeling is not restricted to the covariances in (2) and (3), which follow from the assumption of a uniform power-delay-profile. The covariance of two channel states, shifted in time and frequency, is $\mathbb{E}\left\{h[t, f] h^{*}\left[t \pm \Delta_{T}, f \pm \Delta_{F}\right]\right\}=c_{T}\left[\Delta_{T}\right] c_{F}\left[\Delta_{F}\right]$.

\section{B. CSI Feedback Model}

In our model CSI is impaired by noisy pilot reception, quantization and a delay due to feedback/backhaul latency (see Fig. 2). For derivations later on, we define $\mathbf{h}_{F}[t, f]=$ $\left[h[t, f], \ldots, h\left[t, f-W_{F}+1\right]^{T}\right.$ as the collection of $W_{F}$ channel states in frequency direction. Furthermore, $W_{T}$ consecutive vectors in time direction are combined within $\mathbf{h}[t, f]=$ $\left[\mathbf{h}_{F}[t, f]^{T}, \ldots, \mathbf{h}_{F}\left[t-W_{T}+1, f\right]^{T}\right]^{T}$. In the following, for readability we omit index $f$ when using $\mathbf{h}_{F}[t, f]$ and $\mathbf{h}[t, f]$.

1) Noisy Pilot Reception: For each transmission block consisting of $L$ symbols, $P$ pilots are transmitted per BS antenna with power $\rho$. The received pilot symbols, each disturbed by Gaussian receiver noise $n \sim \mathcal{N}_{\mathbb{C}}\left(0, \sigma_{n}^{2}\right)$, can equivalently be written as $\mathbf{x}=\mathbf{h}_{F}[t]+\mathbf{z}$, introducing the effective Gaussian noise $\mathbf{z} \sim \mathcal{N}_{\mathbb{C}}\left(\mathbf{0}, \sigma_{z}^{2} \mathbf{I}\right)$ with variance $\sigma_{z}^{2}=\sigma_{n}^{2} /\left(P \rho^{2}\right)$ [16]

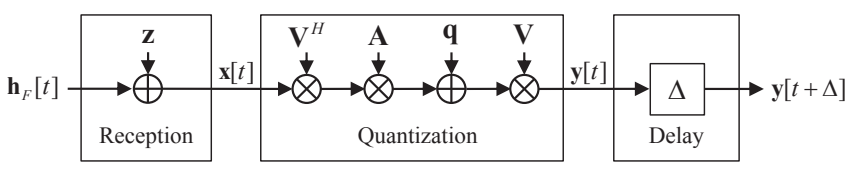

Fig. 2. Feedback model for reporting CSI back to the BS.

2) Quantization: The noisy channel observations $\mathbf{x}=$ $\mathbf{h}_{F}[t]+\mathbf{z}$ are quantized using $Q$ bits followed in order to feed them back to the BS over a limited rate feedback link. While quantization of a large number of i.i.d. Gaussian random variables can be modeled with rate distortion theory [14], [17], the observation vector $\mathrm{x}$ contains realizations of correlated Gaussian random variables. At this point we extend our previous work [14] and de-correlate $\mathrm{x}$ by multiplying with $\mathbf{V}^{H}$, which results from eigen value decomposition of the covariance matrix $\mathbb{E}\left\{\mathbf{x} \mathbf{x}^{H}\right\}=\mathbf{\Phi}_{\mathbf{x}}=\mathbf{V} \boldsymbol{\Sigma} \mathbf{V}^{H}$. Since the components of vector $\mathbf{V}^{H} \mathbf{x}$ have different variances, the total number of $Q$ bits are allocated among the $W_{F}$ uncorrelated components, where element $w$ is quantized with $Q_{w}$ bits, such that $Q=\sum_{w=1}^{W_{F}} Q_{w}$.

The assumption of a large number of i.i.d. coefficients, in order to allow modeling with rate distortion theory, can be motivated by multiple independent transmission block collections $\mathbf{h}_{F}[t]$ distributed over the spectrum and multiple antennas at BS and UE side.

According to the model in [17] and its extension in [14] quantizing a sequence of realizations of a Gaussian distributed random variable $x$ can be written as

$$
y=a x+q,
$$

with scalar $a=1-2^{-Q}$ and additive Gaussian distributed quantization noise $q \sim \mathcal{N}_{\mathbb{C}}\left(0, \sigma_{q}^{2}\right)$ with variance

$$
\sigma_{q}^{2}=2^{-Q}\left(1-2^{-Q}\right) \sigma_{x}^{2}
$$

The adaptation of the model in (4) to the quantization of vector $\mathbf{V}^{H} \mathbf{X}$ with independent entries of different variances results in

$$
\mathbf{y}=\mathbf{A} \mathbf{V}^{H} \mathbf{x}+\mathbf{q}
$$

where $\mathbf{A}=\operatorname{diag}\left(\left[1-2^{-Q_{1}}, \ldots, 1-2^{-Q_{W_{F}}}\right]\right)$ is a diagonal matrix and $\mathbf{q} \sim \mathcal{N}_{\mathbb{C}}\left(\mathbf{0}, \Phi_{\mathbf{q}}\right)$ denotes the quantization noise with covariance matrix $\boldsymbol{\Phi}_{\mathbf{q}}=\mathbf{A}(\mathbf{I}-\mathbf{A}) \mathbf{V}^{H} \boldsymbol{\Phi}_{\mathbf{x}} \mathbf{V}$.

3) Outdated CSI: Outdated CSI is reflected by the correlation between two channel coefficients delayed by $\Delta$ transmission blocks in time, according to (2).

4) Combined Feedback Model: Noisy pilot reception, quantization and outdated CSI can be combined to a single equation. The observations available at the BS with delay $\Delta$ reads

$$
\mathbf{y}[t]=\mathbf{V}\left(\mathbf{A V}^{H}\left(\mathbf{h}_{F}[t-\Delta]+\mathbf{z}\right)+\mathbf{q}\right),
$$

as illustrated in Fig. 2. In order to obtain an estimate of the channel for a certain point in the future, channel prediction can be included into the feedback chain. The different options of employing prediction is described in the next section. 


\section{Channel Prediction}

In this section the MSE between the actual channel and the predicted channel as well as the minimum MSE (MMSE) channel estimate is derived for prediction at the UE (P-UE), prediction at the $\mathrm{BS}(\mathrm{P}-\mathrm{BS})$, and joint prediction at $\mathrm{BS}$ and $\mathrm{UE}$. Having in mind that for distributed precoding CSI is affected by multiple delays due to feedback and backhaul latency, PBS brings the flexibility to predict at each BS individually, according to the actually occurring delay. In contrast, P$\mathrm{UE}$ is restricted to predict for a single point in time. With the feedback model presented in Sec. II multiple channel observations $\mathbf{y}[t]$ can be combined to get a more accurate estimate of $\mathbf{h}_{F}[t]$. For this purpose we use the channel vector $\mathbf{h}[t]$ including consecutive channel states in time.

\section{A. Channel Prediction at the User Equipment}

For that scheme the channel prediction is placed at the UE side before observations are quantized, as illustrated in Fig. 3. The noisy channel observations $\mathbf{x}=\mathbf{h}[t]+\mathbf{z}$ are multiplied with the channel prediction matrix $\mathbf{G}[\Delta]$ intending to compensate for the delay $\Delta$. The resulting transmission equation reads

$$
\hat{\mathbf{h}}_{F}[t]=\mathbf{V}_{U}\left(\mathbf{A}_{U} \mathbf{V}_{U}^{H} \mathbf{G}[\Delta](\mathbf{h}[t-\Delta]+\mathbf{z})+\mathbf{q}_{U}\right) .
$$

The matrix $\mathbf{G}[\Delta]$ is optimized in order to minimizes the MSE between the actual channel vector and its estimate

$$
\begin{aligned}
\epsilon_{U}= & \mathbb{E}\left\{\left(\mathbf{h}_{F}[t]-\hat{\mathbf{h}}_{F}[t]\right)\left(\mathbf{h}_{F}[t]-\hat{\mathbf{h}}_{F}[t]\right)^{H}\right\} \\
= & W_{F}+\operatorname{tr}\left(\mathbf{V}_{U} \mathbf{A}_{U} \mathbf{V}_{U}^{H} \mathbf{G}[\Delta]\left(\mathbf{C}+\sigma_{z}^{2} \mathbf{I}\right) \mathbf{G}^{H}[\Delta]\right) \\
& -\operatorname{tr}\left(\mathbf{V}_{U} \mathbf{A}_{U} \mathbf{V}_{U}^{H} \mathbf{G}[\Delta] \overline{\mathbf{C}}[\Delta]\right) \\
& -\operatorname{tr}\left(\overline{\mathbf{C}}[\Delta]^{H} \mathbf{G}[\Delta]^{H} \mathbf{V}_{U} \mathbf{A}_{U} \mathbf{V}_{U}^{H}\right) .
\end{aligned}
$$

with $\mathbf{C}=\mathbb{E}\left\{\mathbf{h}[t] \mathbf{h}^{H}[t]\right\}$ and $\overline{\mathbf{C}}[\Delta]=\mathbb{E}\left\{\mathbf{h}[t] \mathbf{h}_{F}^{H}[t+\Delta]\right\}$, while the quantization is adapted according to $\mathbf{G}\left(\mathbf{C}+\sigma_{z}^{2} \mathbf{I}\right) \mathbf{G}^{H}=$ $\mathbf{V}_{U} \boldsymbol{\Sigma}_{U} \mathbf{V}_{U}^{H}$ and $\boldsymbol{\Phi}_{\mathbf{q}_{U}}=\mathbf{A}(\mathbf{I}-\mathbf{A}) \mathbf{V}_{U}^{H} \mathbf{G}\left(\mathbf{C}+\sigma_{z}^{2} \mathbf{I}\right) \mathbf{G}^{H} \mathbf{V}_{U}$ The MMSE channel prediction matrix is obtained by setting the derivative of (9) with respect to $\mathbf{G}[\Delta]$ equal to zero. Based on the Wirtinger derivations we obtain

$$
\begin{aligned}
\frac{\partial \epsilon}{\partial \mathbf{G}[\Delta]}= & \mathbf{V}_{U}^{*} \mathbf{A}_{U}^{T} \mathbf{V}_{U}^{T} \mathbf{G}[\Delta]^{*}\left(\mathbf{C}+\sigma_{z}^{2} \mathbf{I}\right)^{T} \\
& -\mathbf{V}_{U}^{*} \mathbf{A}_{U}^{T} \mathbf{V}_{U}^{T} \overline{\mathbf{C}}[\Delta]^{T} \stackrel{!}{=} \mathbf{0}
\end{aligned}
$$

Rearranging (10) leads to the MMSE channel prediction matrix

$$
\mathbf{G}[\Delta]=\overline{\mathbf{C}}^{H}[\Delta]\left(\mathbf{C}+\sigma_{z}^{2} \mathbf{I}\right)^{-1} .
$$

Note, that the predictor (11) is independent of the quantizer resolution, since the scaling with $\mathbf{A}$ inherently assesses the quality of the quantizer outcome [14]. The MSE obtained by inserting (11) into (9) results in

$$
\epsilon_{U}=W_{F}-\operatorname{tr}\left(\mathbf{V}_{U} \mathbf{A}_{U} \mathbf{V}_{U}^{H} \overline{\mathbf{C}}^{H}[\Delta]\left(\mathbf{C}+\sigma_{z}^{2} \mathbf{I}\right)^{-1} \overline{\mathbf{C}}[\Delta]\right),
$$

where (12) is the sum MSE over all $W_{F}$ subcarriers.

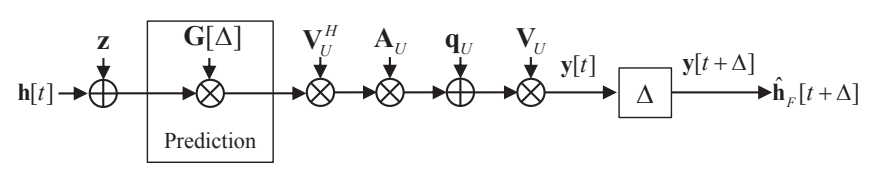

Fig. 3. Channel feedback chain with prediction at the UE (P-UE)

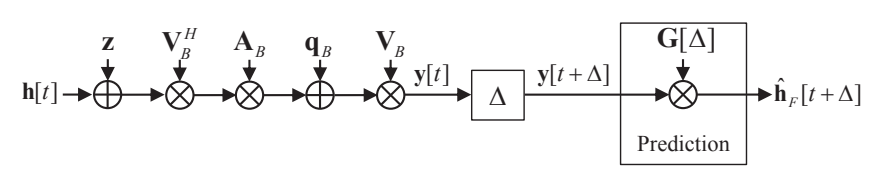

Fig. 4. Channel feedback chain with prediction at the BS (P-BS)

\section{B. Channel Prediction at the Base Station}

Now the channel prediction matrix is placed at the BS side, as illustrated in Fig. 4. Here the outdated channel observations $\mathbf{y}[t+\Delta]$ available at the BS are multiplied with $\mathbf{G}[\Delta]$ in order to compensate for the delay $\Delta$. The respective transmission equation results in

$$
\hat{\mathbf{h}}_{F}[t]=\mathbf{G}[\Delta] \mathbf{V}_{B}\left(\mathbf{A}_{B} \mathbf{V}_{B}^{H}(\mathbf{h}[t-\Delta]+\mathbf{z})+\mathbf{q}_{B}\right) .
$$

In this model we assume that in each time instance $t$ a single frame $\mathbf{h}_{F}[t]$ is reported to the BS, i.e., $\mathbf{h}[t]$ is transmitted via $W_{T}$ consecutive time instances, where the de-correlation and quantization is the same for all $W_{T}$ transmissions. Consequently, the de-correlation matrix $\mathbf{V}_{B}=\mathbf{I} \otimes \mathbf{V}$ has identical blocks $\mathbf{V}$ on its diagonal, where $\otimes$ is the Kronicker product. Also the scaling matrix $\mathbf{A}_{B}=\mathbf{I} \otimes \mathbf{A}$ consists of equal blocks A on its diagonal. Hence, the vector $\mathbf{y}[t+\Delta]$ consists of $W_{T}$ consecutive channel observations, where each of them is identically and independently processed. Only at the BS the $W_{T}$ observations are combined by employing channel prediction. Corresponding to Sec. III-A, we proceed by calculating the derivative of the MSE

$$
\begin{aligned}
\frac{\partial \epsilon}{\partial \mathbf{G}[\Delta]}= & \mathbf{G}[\Delta]^{*}\left(\mathbf{C}+\sigma_{z}^{2} \mathbf{I}\right)^{T} \mathbf{V}_{B}^{*} \mathbf{A}_{B}^{T} \mathbf{V}_{B}^{T} \\
& -\overline{\mathbf{C}}[\Delta]^{T} \mathbf{V}_{B}^{*} \mathbf{A}_{B}^{T} \mathbf{V}_{B}^{T} \stackrel{!}{=} \mathbf{0} .
\end{aligned}
$$

We see that the MMSE channel predictor for P-BS is equivalent with the MMSE predictor for P-UE in (11). A similar relation was found in our previous work [14] considering uncorrelated subcarriers only. The MSE obtained by inserting $\mathbf{G}[\Delta]$ results in

$$
\epsilon_{B}=W_{F}-\operatorname{tr}\left(\mathbf{V}_{B} \mathbf{A}_{B} \mathbf{V}_{B}^{H} \overline{\mathbf{C}}[\Delta] \overline{\mathbf{C}}^{H}[\Delta]\left(\mathbf{C}+\sigma_{z}^{2} \mathbf{I}\right)^{-1}\right) .
$$

\section{Equivalence of $P-U E$ and $P-B S$}

In this section we show that the MSE (12) for P-UE is equivalent with the MSE for P-BS (15). We first make use of the transformation

$$
\operatorname{tr}(\mathrm{AD})=\mathbf{a}^{T} \mathbf{d},
$$

for a diagonal matrix $\mathbf{A} \in \mathbb{C}^{N \times N}$ and an arbitrary quadratic matrix $\mathbf{D} \in \mathbb{C}^{N \times N}$, where $\mathbf{a}=\operatorname{diag}^{-1}(\mathbf{A}) \in \mathbf{C}^{N \times 1}$ and $\mathbf{d}=\operatorname{diag}^{-1}(\mathbf{D}) \in \mathbf{C}^{N \times 1}$. Since matrices can be rotated within the trace function, with $\mathbf{a}_{U}=\operatorname{diag}^{-1}\left(\mathbf{A}_{U}\right)$ and 
$\mathbf{a}_{B}=\operatorname{diag}^{-1}\left(\mathbf{A}_{B}\right)$ as well as

$$
\mathbf{d}_{U}=\operatorname{diag}^{-1}\left(\mathbf{V}_{U}^{H} \overline{\mathbf{C}}^{H}[\Delta]\left(\mathbf{C}+\sigma_{z}^{2} \mathbf{I}\right)^{-1} \overline{\mathbf{C}}[\Delta] \mathbf{V}_{U}\right)
$$

and

$$
\mathbf{d}_{B}=\operatorname{diag}^{-1}\left(\mathbf{V}_{B}^{H} \overline{\mathbf{C}}[\Delta] \overline{\mathbf{C}}^{H}[\Delta]\left(\mathbf{C}+\sigma_{z}^{2} \mathbf{I}\right)^{-1} \mathbf{V}_{B}\right)
$$

we can rewrite the MSEs of (12) and (15) as $\epsilon_{U}=W_{F}-\mathbf{a}_{U}^{T} \mathbf{d}_{U}$ and $\epsilon_{B}=W_{F}-\mathbf{a}_{B}^{T} \mathbf{d}_{B}$. Based on $\mathbf{d}_{U}=\left[d_{U, 1}, \ldots, d_{U, W_{F}}\right]^{T}$ and $\mathbf{d}_{B}=\left[d_{B, 1}, \ldots, d_{B, W_{F}}, d_{B, 1+W_{F}}, \ldots, d_{B, W_{F} W_{T}}\right]^{T}$ the MSEs result result in

$$
\epsilon_{U}=W_{F}+\sum_{l=1}^{W_{F}}\left(2^{-Q_{U, l}}-1\right) d_{U, l}
$$

and

$$
\epsilon_{B}=W_{F}+\sum_{l=1}^{W_{F}}\left(2^{-Q_{B, l}}-1\right) \sum_{k=1}^{W_{T}} d_{B, l+(k-1) W_{T}} .
$$

The $2^{-Q_{B, l}}$ in (15) can be excluded since the processing is equivalent at each time instance, i.e., for each $k W_{T^{-}}$th component of vector $\mathbf{h}[t]$. With

$$
d_{U, l}=\sum_{k=1}^{W_{T}} d_{B, l+(k-1) W_{T}}
$$

it is shown that (19) and (20) are equivalent and prediction before and after quantization results in the same CSI quality. That (21) holds, can be found numerically, while a detailed derivation is skipped at this point, due to space issues.

\section{Joint Channel Prediction}

In this section channel prediction is placed at both, UE and BS side. In order to have a fair comparison with the previous sections, the overall processing window need to be equivalent. Hence, the channel estimate $\hat{\mathbf{h}}_{F}[t]$ need to be obtained only based on observations of $\mathbf{h}[t-\Delta]$. Based on that, the channel predictor at the $\mathrm{UE}_{U}[\Delta]$ has block diagonal structure, with equivalent blocks on the diagonal. By placing an additional prediction matrix $\mathbf{G}_{B}[\Delta]$ at the $\mathrm{BS}$ side we obtain the following transmission equation

$$
\hat{\mathbf{h}}_{F}[t]=\mathbf{G}_{B}[\Delta] \mathbf{V}_{J}\left(\mathbf{A}_{J} \mathbf{V}_{J}^{H} \mathbf{G}_{U}[\Delta](\mathbf{h}[t-\Delta]+\mathbf{z})+\mathbf{q}_{J}\right) .
$$

According to the methodology of the previous sections the MMSE channel predictor $\mathbf{G}_{B}[\Delta]$ applied at the BS results in

$$
\mathbf{G}_{B}[\Delta]=\overline{\mathbf{C}}^{H}[\Delta] \mathbf{G}_{U}^{H}[\Delta]\left(\mathbf{G}_{U}[\Delta]\left(\mathbf{C}+\sigma_{z}^{2} \mathbf{I}\right) \mathbf{G}_{U}^{H}[\Delta]\right)^{-1} .
$$

The MSE obtained by including $\mathbf{G}_{B}[\Delta]$ results in

$$
\begin{aligned}
\epsilon_{J}= & W_{F}-\operatorname{tr}\left(\overline{\mathbf{C}}^{H}[\Delta] \mathbf{G}_{U}^{H}[\Delta] \mathbf{V}_{J} \mathbf{A}_{J} \mathbf{V}_{J}^{H} .\right. \\
& \left.\cdot\left(\mathbf{G}_{U}[\Delta]\left(\mathbf{C}+\sigma_{z}^{2} \mathbf{I}\right) \mathbf{G}_{U}^{H}[\Delta]\right)^{-1} \mathbf{G}_{U}[\Delta] \overline{\mathbf{C}}[\Delta]\right) .
\end{aligned}
$$

By comparing (24) with (15) we observe, that we cannot find any matrix $\mathbf{G}_{U}$ such that $\epsilon_{J}<\epsilon_{U}=\epsilon_{B}$. Consequently, prediction at both, UE and BS side does not result in any additional performance gains.

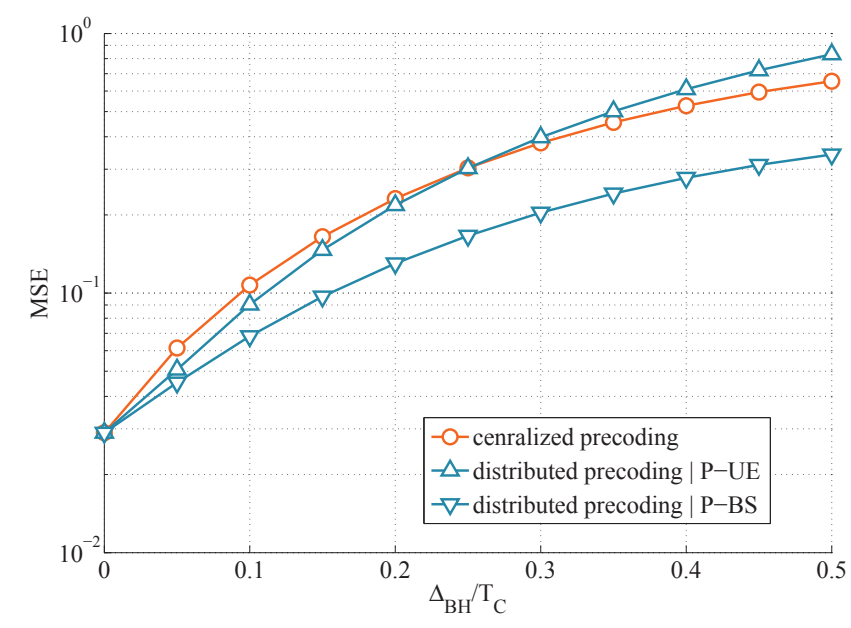

Fig. 5. Average user MSE over the backhaul delay $\Delta_{B H}$ normalized to the coherence time $T_{C}$.

\section{RESUlts}

We illustrate our findings by a sandbox scenario with 2 UEs jointly served by $2 \mathrm{BSs}$, according to Fig. 1. The delay for feedback transmission is $\Delta_{F B}=0.05 T_{C}$, where $T_{C}$ is the $50 \%$ coherence time. In Fig. 5 we plotted the average user MSE for different strategies. For centralized precoding, CSI of both UEs is affected by $\Delta_{F B}+\Delta_{B H}$. For distributed precoding with P-UE, the prediction compensates for $\Delta_{F B}$, while the CSI of the UE located in the other cell is affected by $\Delta_{F B}+\Delta_{B H}$. For P-BS the prediction always compensates for the actually occurring delay, resulting in the best CSI accuracy. Since the basic statement of this paper is that the equivalence of prediction before and after quantization also holds for the general case of correlated subcarriers, we skip more detailed results on the downlink performance and refer to [14].

\section{CONCLUSIONS}

In this work, we analyzed different prediction options for a cooperative multi-cell setup, where CSI is impaired by channel estimation errors, quantization and feedback/backhaul delays. We extend our previous work to the more general case of correlated subcarriers. We showed, that placing channel prediction before the quantizer at the UE results in the same CSI accuracy as predicting the channel based on quantized observations at the BS. The same relation we already found for un-correlated subcarriers. Applying this finding to a cooperative multi-cell setup, we showed the average channel MSE of two UEs assigned to different BSs. Distributed precoding with channel prediction at the BS results in the highest CSI accuracy, while prediction at the UE leads to mismatched compensation for the UE of the other cell. Furthermore, it was shown that centralized precoding performs similar to prediction at the UE, all CSI is affected by the backhaul delay.

\section{ACKNOWLEDGEMENT}

This work was supported by the German Science Foundation (DFG) within the priority program COIN under project grant $\mathrm{Fe}$ 423/12-2. 


\section{REFERENCES}

[1] S. Shamai and B. Zaidel, "Enhancing the Cellular Downlink Capacity via Co-Processing at the Transmitting End," in Proc. IEEE Vehicular Technology Conference (VTC 'O1-Spring), 2001.

[2] H. Zhang and H. Dai, "Cochannel Interference Mitigation and Cooperative Processing in Downlink Multicell Multiuser MIMO Networks,' EURASIP Journal on Wireless Communications and Networking, no. 2, pp. 222-235, 2004.

[3] M. Karakayali, G. Foschini, and R. Valenzuela, "Network Coordination for Spectrally Efficient Communications in Cellular Systems," IEEE Transactions on Wireless Communications, vol. 13, no. 4, pp. $56-61$, 2006.

[4] S. Kaviani, O. Simeone, W. Krzymien, and S. Shamai, "Linear Precoding and Equalization for Network MIMO With Partial Cooperation," IEEE Transactions on Vehicular Technology, vol. 61, no. 5, pp. 2083-2096, 2012.

[5] R. Fritzsche and G. Fettweis, "Robust Precoding with General Power Constraints Considering Unbounded Channel Uncertainty," in Proc. IEEE International Symposium on Wireless Communication Systems (ISWCS '12), 2012.

[6] N. Jindal, "MIMO Broadcast Channels with Finite Rate Feedback," in Proc. IEEE Global Telecommunications Conference (GLOBECOM '05), 2005.

[7] P. Castro, M. Joham, L. Castedo, and W. Utschick, "Optimized CSI Feedback for Robust THP Design," in Proc. Asilomar Conference on Signals, Systems and Computers, 2007. (ACSSC '07)., 2007.

[8] D. Love, R. Heath, V. Lau, D. Gesbert, B. Rao, and M. Andrews, "An overview of limited feedback in wireless communication systems," IEEE Journal on Selected Areas in Communications, vol. 26, no. 8, pp. 13411365, 2008.

[9] P. M. Castro, M. Joham, L. Castedo, and W. Utschick, "Robust MMSE linear precoding for Multiuser MISO systems with limited feedback and channel prediction," in Proc. IEEE International Symposium on Personal, Indoor and Mobile Radio Communications (PIMRC '08), 2008.

[10] R. Fritzsche and G. Fettweis, "Robust Sum Rate Maximization in the Multi-Cell MU-MIMO Downlink," in Proc. IEEE Wireless Communications and Networking Conference (WCNC '13), 2013.

[11] A. Papadogiannis, E. Hardouin, and D. Gesbert, "Decentralising Multicell Cooperative Processing: A Novel Robust Framework," EURASIP Journal on Wireless Communications and Networking, vol. 2009, pp. $1-11,2009$.

[12] R. Fritzsche and G. Fettweis, "CSI Distribution for Joint Processing in Cooperative Cellular Networks," in Proc. IEEE Vehicular Technology Conference (VTC '11-Fall), 2011.

[13] R. Zhang and L. Hanzo, "Joint and Distributed Linear Precoding for Centralised and Decentralised Multicell Processing," in Proc. IEEE Vehicular Technology Conference Fall (VTC '10-Fall), 2010.

[14] R. Fritzsche, E. Ohlmer, and G. Fettweis, "Where to Predict the Channel in Cooperative Cellular Networks with Backhaul Delay?" in Proc. IEEE International Conference on Systems, Communications and Coding (SCC '13), 2013.

[15] P. Hoeher, S. Kaiser, and P. Robertson, "Two-Dimensional Pilot-SymbolAided Channel Estimation by Wiener Filtering," in Proc. IEEE International Conference on Acoustics, Speech, and Signal Processing (ICASSP '97), 1997.

[16] S. M. Kay, Fundamentals of Signal Processing: Estimation Theory, 1st ed. Prentice Hall PTR, 1993.

[17] T. M. Cover and J. A. Thomas, Elements of Information Theory, 2nd ed. John Wiley and Sons, 2006. 